Original Article

\title{
Kinematic head and trunk strategies used by hemiplegic stroke patients crossing over obstacles of different heights
}

\author{
Jin-Tae Han, PhD, PT ${ }^{1)}$, Jung-Hoon Lee, PhD, PT ${ }^{2 *}$, Dennis W. Fell, MD, PT ${ }^{3)}$ \\ 1) Department of Physical Therapy, College of Science, Kyung-sung University, Republic of Korea \\ 2) Department of Physical Therapy, College of Nursing and Healthcare Sciences, Dong-Eui University: \\ 176 Eomgwangno, Busanjin-gu, Busan 614-714, Republic of Korea \\ 3) Department of Physical Therapy, University of South Alabama, USA
}

\begin{abstract}
Purpose] The purpose of this study was to compare kinematic data regarding the head, trunk, and pelvis strategies used by individuals with hemiplegia when crossing over obstacles of different heights. [Subjects and Methods] Nine adults with hemiplegia from stroke ( 7 males and 2 females) participated in this study. A motion analysis system with six infrared cameras was used to measure the kinematic data of the head, trunk, and pelvis while the subjects crossed over obstacles of different heights. Repeated measures ANOVA analysis was performed to compare the resulting kinematic data. [Results] An increase in the magnitude of the kinematic data of the head, trunk, and pelvis of the hemiparetic stroke patients was observed when the height of the obstacles, which they crossed over, increased. [Conclusion] This study described the kinematic strategies, with regard to the head, trunk, and pelvis, used by hemiplegic patients crossing over obstacles of different heights. The results indicate that these kinematic strategies primarily change when the obstacle height was $20 \%$ of the height of the subjects. Key words: Stroke, Trunk, Obstacle
\end{abstract}

(This article was submitted Sep. 1, 2016, and was accepted Oct. 11, 2016)

\section{INTRODUCTION}

Crossing over obstacles is one of many tasks in daily life that is associated with ambulation ${ }^{1)}$. For stroke patients, safely crossing over an obstacle is a challenging task ${ }^{2}$, and the probability of failure is very high ${ }^{3)}$. It is known that nearly $50 \%$ of stroke patients fail to cross over an obstacle due to their difficulty with maintaining balance ${ }^{2)}$. A previous study showed that $10 \%$ of falls by stroke patients who were discharged from hospital were related to obstacles ${ }^{4}$, and another study showed that 13 out of 24 stroke patients who could walk without physical assistance, or with a gait-assisting instrument, had difficulty crossing over even small obstacles ${ }^{2}$.

It is known that when stroke patients cross over an obstacle, their walking slows down, and their gait pattern changes, exhibiting alterations in the trajectory of the lead limb, which is the first limb used to cross over the obstacle ${ }^{3)}$. It has also been reported that subjects experienced difficulty with crossing over a high obstacle $(1-8 \mathrm{~cm})$ and required an assisting instrument or a rail to keep their balance ${ }^{1)}$. Thus, understanding the extent and distinctive features of post-stroke during obstacle-crossing is important for identifying the risks associated with stroke patients' movements and for establishing a treatment strategy ${ }^{1)}$. However, most previous studies of stroke patients walking have only involved an evaluation of stroke patients' gait performance or balance ability after treatment intervention ${ }^{5-7)}$; studies that have collected kinematic data of body areas, such as the head, trunk, and pelvis of stroke patients crossing over obstacles of different heights are few. Thus, the purpose of this study was to compare the kinematics of the head, trunk, and pelvis of individuals with hemiplegia stepping 
Table 1. Characteristics of the subjects

\begin{tabular}{ccccccc}
\hline Subject & Gender & Age (yrs) & $\begin{array}{c}\text { Time since } \\
\text { stroke (months) }\end{array}$ & $\begin{array}{c}\text { Involved } \\
\text { side }\end{array}$ & Stroke type & FAC \\
\hline 1 & M & 47 & 8 & Right & Ischemic & Grade 4 \\
2 & M & 65 & 31 & Left & Hemorrhagic & Grade 3 \\
3 & M & 21 & 18 & Left & Hemorrhagic & Grade 4 \\
4 & M & 48 & 31 & Left & Hemorrhagic & Grade 4 \\
5 & M & 37 & 28 & Left & Hemorrhagic & Grade 4 \\
6 & M & 67 & 22 & Left & Ischemic & Grade 4 \\
7 & M & 38 & 30 & Right & Hemorrhagic & Grade 4 \\
8 & F & 46 & 30 & Right & Ischemic & Grade 4 \\
9 & F & 49 & 26 & Right & Hemorrhagic & Grade 3 \\
\hline
\end{tabular}

M: male; F: female; FAC: functional ambulation category scale

over obstacles of different heights.

\section{SUBJECTS AND METHODS}

Nine adults with hemiplegia from stroke ( 7 males and 2 females) participated in this study. The subjects had a mean \pm SD age of $46.4 \pm 14.1$ years, weight of $68.4 \pm 10.8 \mathrm{~kg}$, height of $170.5 \pm 13.6 \mathrm{~cm}$, leg length of $86.12 \pm 5.38 \mathrm{~cm}, \mathrm{knee}$ width of $10.02 \pm 1.45 \mathrm{~cm}$, and ankle width of $8.22 \pm 1.84 \mathrm{~cm}$. Subjects with hemiparesis (paretic side: 5 right, 4 left), who were able to walk for at least 5 minutes without walking aid tools (FAC grade: 3-4), were recruited by a medical doctor at a community-based rehabilitation hospital. Prior to the start of the study, each patient provided a written informed consent document that was approved by the National Evidence-based Healthcare Collaborating Agency. This study conformed to the ethical principles of the Declaration of Helsinki.

A motion analysis system with six infrared cameras $(60 \mathrm{~Hz}$, Hawk Digital System, Motion Analysis Corporation, Santa Rosa, CA, USA) was used to measure the kinematic data of the head, trunk, and pelvis while the subjects crossed over obstacles of different heights. Twenty-four reflective markers were attached to the subjects' bodies. The markers were placed on the head, trunk, pelvis, thighs, shanks, feet, and arms. The amplified motion analysis signals were sampled on-line using EVaRT 5.0 software and analyzed using Cortex 64 and OrthoTrak 6.6.4 software. All subjects were asked to walk barefoot at a self-selected pace on an 8-m walkway. Self-made obstacles (width: $1.5 \mathrm{~m}$, height: $50 \mathrm{~cm}$ ) with an adjustable height were used. Subjects were asked to step over obstacles of different heights in a random order. The height of the obstacle was adjusted to $0 \%, 10 \%$, and $20 \%$ of the subject's height. Two walking trials were performed in consideration of the subject's fatigue, and the mean of the value of the two trials was used in the analysis. Prior to ensemble averaging, individual data were normalized with respect to the time of the $0-100 \%$ gait cycle. This study measured the angles of the head, trunk, and pelvis in three dimensions in the swing phase of the patients' paretic limbs while they stepped over obstacles of different heights.

Repeated measures ANOVA analysis was performed to compare the kinematics of the head, trunk, and pelvis while crossing over the obstacles of different heights. All statistical analyses were performed using SPSS ver. 21.0 (IBM Inc., Chicago, IL, USA) and $\mathrm{p}$ values less than 0.05 were considered significantly different.

\section{RESULTS}

The general characteristics of the subjects are shown in Table 1. When subjects with paretic limbs crossed over obstacles that were $10 \%$ of their height, the magnitude of the kinematic data of the subject' head, trunk, and pelvis did not increase significantly, compared to that recorded when there was no obstacle ( $\mathrm{p}>0.05)$ (Table 2).

The subjects' maximum forward flexion of the head while crossing over an obstacle that was $20 \%$ of their height was significantly increased compared to that when there was no obstacle $(\mathrm{p}<0.05)$. The subjects' maximum lateral flexion of the head and the maximum rotation of the pelvis of their non-paretic side while crossing over an obstacle that was $20 \%$ of their height significantly increased compared to their respective values when the height of the obstacles was $10 \%$ of their height $(\mathrm{p}<0.05)$ (Table 2).

\section{DISCUSSION}

An increase in the magnitude of the kinematic data of the head, trunk, and pelvis of individuals with paretic limbs was observed when the height of the obstacle the individuals crossed over increased. These increases were considered to be compensatory movement strategies used by the head, trunk, and pelvis to raise the swing limb elevation when the height of 
Table 2. Comparison of kinematic data of the head, trunk, and pelvis of hemiparetic stroke patients stepping over obstacles of different heights (mean $\pm \mathrm{SD}$ )

\begin{tabular}{llccc}
\hline Body & Variables & $0 \%$ Height & $10 \%$ Height & $20 \%$ Height \\
\hline \multirow{4}{*}{ Head } & Max. lateral flex of NP side & $4.33 \pm 2.49$ & $4.51 \pm 1.75$ & $7.52 \pm 3.54^{*} \dagger$ \\
& Max. forward flex & $17.40 \pm 10.98$ & $25.47 \pm 11.99$ & $35.91 \pm 11.73^{*}$ \\
& Max. rotation of NP side & $2.42 \pm 1.96$ & $3.70 \pm 1.93$ & $5.84 \pm 2.35^{*}$ \\
\multirow{3}{*}{ Trunk } & Max. lateral flex & $1.41 \pm 0.97$ & $3.18 \pm 2.79$ & $6.00 \pm 4.29$ \\
& Max. forward flex & $2.55 \pm 1.22$ & $4.22 \pm 2.95$ & $5.30 \pm 4.65$ \\
& Max. rotation f NP side & $1.64 \pm 1.32$ & $2.98 \pm 1.36$ & $4.85 \pm 2.93^{*}$ \\
& Max. obliquity f NP side & $3.10 \pm 1.76$ & $6.19 \pm 3.78$ & $12.41 \pm 6.38^{*}$ \\
Pelvis & Max. AP tilt & $12.22 \pm 2.27$ & $18.62 \pm 6.69$ & $21.02 \pm 8.49$ \\
& Max. rotation f NP side & $1.69 \pm 1.09$ & $2.87 \pm 1.96$ & $10.72 \pm 4.90^{* \dagger}$ \\
\hline
\end{tabular}

NP: non-paretic; AP: anteroposterior

$* \mathrm{p}<0.05$ : significant difference between the $0 \%$ and $20 \%$ height obstacles

$\dagger \mathrm{p}<0.05$ : significant difference between the $10 \%$ and $20 \%$ height obstacles

the obstacle increased, and also to reduce the risk of falling by enhancing the stability of the non-paretic side.

When crossing over an obstacle, increased swing limb elevation increases balance demands ${ }^{8)}$. In addition, stroke patients adopt various strategies to enhance their balance ability when crossing over an obstacle ${ }^{3}$. As the height of the obstacle increases, elevation of the swing limb also increases, and stroke patients aim to secure greater stability by shifting their center of gravity (COG) toward their non-paretic side. They try achieving this through lateral flexion and rotation of the head and the trunk, as well as through obliquity and rotation of the pelvis. Further, stroke patients try lowering their overall height through forward flexion of the head and trunk. It is also assumed that stroke patients try to reduce the risk of fall by lowering their COG. The results of this study indicate that these kinematic strategies primarily changed when the subjects crossed over obstacles that were $20 \%$ of their height.

This study had several limitations. First, kinematic data of other joints, such as the knee and ankle were not recorded. Second, the number of subjects was too small to generalize the research findings. Third, changes in the kinematic data due to differences in the depth of the obstacles were not examined. Fourth, the muscle activities of the related muscles while subjects crossed over the obstacles were not analyzed. Thus, follow-up studies are needed to address these issues.

\section{REFERENCES}

1) Said CM, Goldie PA, Patla AE, et al.: Effect of stroke on step characteristics of obstacle crossing. Arch Phys Med Rehabil, 2001, 82: 1712-1719. [Medline] [CrossRef]

2) Said CM, Goldie PA, Patla AE, et al.: Obstacle crossing in subjects with stroke. Arch Phys Med Rehabil, 1999, 80: 1054-1059. [Medline] [CrossRef]

3) Said CM, Goldie PA, Patla AE, et al.: Balance during obstacle crossing following stroke. Gait Posture, 2008, 27: 23-30. [Medline] [CrossRef]

4) Forster A, Young J: Incidence and consequences of falls due to stroke: a systematic inquiry. BMJ, 1995, 311: 83-86. [Medline] [CrossRef]

5) Hung JW, Chen PC, Yu MY, et al.: Long-term effect of an anterior ankle-foot orthosis on functional walking ability of chronic stroke patients. Am J Phys Med Rehabil, 2011, 90: 8-16. [Medline] [CrossRef]

6) Kang TW, Lee JH, Cynn HS: Six-week Nordic treadmill training compared with treadmill training on balance, gait, and activities of daily living for stroke patients: a randomized controlled trial. J Stroke Cerebrovasc Dis, 2016, 25: 848-856. [Medline] [CrossRef]

7) Park JH, Hwangbo G, Kim JS: The effect of treadmill-based incremental leg weight loading training on the balance of stroke patients. J Phys Ther Sci, 2014, 26: 235-237. [Medline] [CrossRef]

8) Chou LS, Kaufman KR, Brey RH, et al.: Motion of the whole body's center of mass when stepping over obstacles of different heights. Gait Posture, 2001, 13: 17-26. [Medline] [CrossRef] 